\title{
Structure of Nascent Microbial Cellulose III. Different Influence of Direct Red 75 and 79 on Nascent Microbial Cellulose
}

\author{
Akira $\mathrm{KAI}^{\dagger}$ and Md. Ibrahim H. MondaL ${ }^{\dagger \dagger}$ \\ Department of Industrial Chemistry, Tokyo Metropolitan University, \\ Minami-Ohsawa, Hachioji, Tokyo 192-0369, Japan
}

(Received October 3, 1997)

\begin{abstract}
The influence of Direct Red 75 and 79 having the same ureylenebis(naphthylazo)benzene skeletal structure on the nascent structure of microbial cellulose was examined. Each product obtained from Acetobacter-culture in the presence of Direct Red 75 or Direct Red 79 is a crystalline complex in which the monolayer of a dye is included between the cellulose sheets corresponding to the (11̄0) plane of microbial cellulose. Cellulose regenerated from each product forms cellulose II. Although the substituent groups in the skeletal structure of both the dyes are not the same, there is no different interactions on the nascent structure of microbial cellulose. Due to the ureylene group on the skeletal structure of dye, stronger hydrogen bonding is occurred between the dye and cellulose in the product. The ureylene group affects stable hydrogen bonding in the cellulose regenerated from Direct Red 75 and Direct Red 79 products.

KEY WORDS Acetobacter xylinum / Dye-Cellulose Complex / Cellulose Sheet / Regenerated Cellulose II / Direct Red 75 / Direct Red 79 /
\end{abstract}

When cellulose producing bacterium, Acetobacter xylinum was cultured in the presence of Direct Red 28 (DR28), a crystalline complex composed of the dye and cellulose was produced and cellulose regenerated from this product formed cellulose $\mathrm{IV}_{\mathrm{I}}{ }^{1} \mathrm{In}$ contrast, although the products in the presence of Direct Blue 1, 14, 15, and 53 have the same biphenylenebis(azo) skeletal structure of DR28, but more two sulfonate, hydroxyl, methoxy or methyl groups on the skeletal structure also formed crystalline complex-like DR28 complex and celluloses regenerated from these complexes were cellulose II. ${ }^{-4}$

Earlier papers ${ }^{1-4}$ report the influence of direct dyes having biphenylenebis(azo) skeletal backbone, but different substituent groups, on the structure of nascent microbial cellulose (MC). The structure of the product as well as the cellulose regenerated was influenced by the number and position of sulfonate groups in a dye. The effects of other substituent groups were not observed.

In this study, to promote a more understanding about the formation of structure of nascent MC, we examine the influence of Direct Red 75 (DR75) and Direct Red 79 (DR79) both having ureylenebis(naphthylazo)benzene skeletal structure on the nascent MC. The difference between these two dyes are substituent groups on the skeletal structure: DR79 has more two methyl and methoxy groups but no amino groups in comparison with DR75.

\section{EXPERIMENTAL}

\section{Materials}

Product Samples. DR75 (1,1'-ureylenebis[3-sulfo-4-(2amino-8-hydroxy-6-sulfo-1-naphthylazo)benzene] tetrasodium salt; Aldrich Chemical Co.) and DR79 (1,1'ureylenebis[2-methoxy-5-methyl-4-(8-hydroxy-3,6-di- sulfo-1-naphthylazo)benzene] tetrasodium salt; Aldrich Chemical Co.), were used as dyes. The product samples (products) were prepared by the culture method described in previous papers. ${ }^{1-4} 60 \mathrm{ml}$ of cellulose-free cell suspension (A. xylinum, IFO 13693) were added to 140 $\mathrm{ml}$ of Hestrin-Schramm's medium (pH 7.0) containing a dye at $0.1 \mathrm{wt} \%$, and the mixture was incubated at $28^{\circ} \mathrm{C}$ for $24 \mathrm{~h}$. To remove the dye not related to dyeing of cellulose, the product collected from the Acetobacterculture in the presence each DR75 or DR79 was rinsed only with distilled water for $1 \mathrm{~h}$. The products were preserved at about $3^{\circ} \mathrm{C}$ in the wet state for the next experiments.

Regeneration of Cellulose from Product Sample. Nondried product samples were subjected to dye-extraction by boiling in $70 \mathrm{vol} \%$ aqueous ethanol for $18 \mathrm{~h}$ (fresh aqueous ethanol solution was used for every $3 \mathrm{~h}$ ). The residual samples were further boiled in a $1.0 \mathrm{wt} \%$ aqueous $\mathrm{NaOH}$ solution, neutralized with $1.0 \mathrm{vol} \%$ aqueous acetic acid and repeatedly rinsed with distilled water. This regenerated cellulose was preserved at $3^{\circ} \mathrm{C}$ in the wet state.
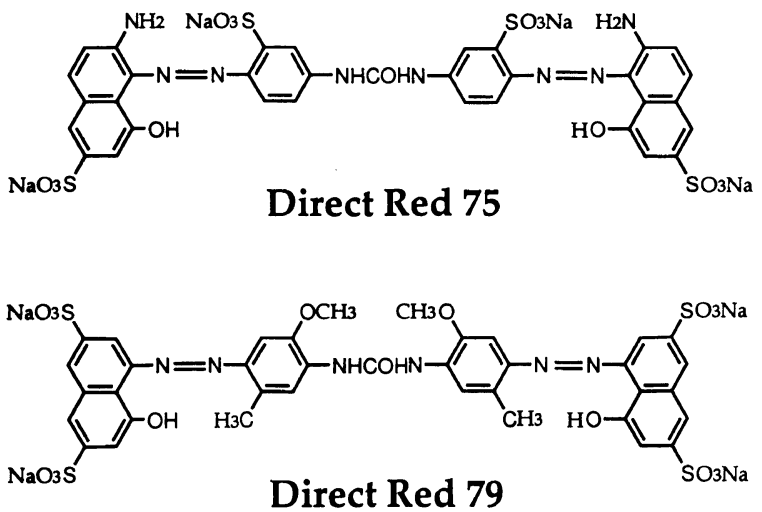

Figure 1. Chemical structures of direct dyes.

\footnotetext{
${ }^{\dagger}$ To whom correspondence should be addressed.

${ }^{\dagger \dagger}$ Present address: Department of Applied Chemistry and Chemical Technology, Rajshahi University, Rajshahi, Bangladesh.
} 
Standard Samples of Cellulose I and II. A standard cellulose I sample was obtained from MC, which after washing with $0.1 \mathrm{wt} \% \mathrm{NaOH}$ solution was boiled in $1.0 \mathrm{wt} \%$ aqueous $\mathrm{NaOH}$ solution for $10 \mathrm{~h}$ under $\mathrm{N}_{2}$ atmosphere, neutralized with $1.0 \mathrm{vol} \%$ aqueous acetic acid solution and repeatedly rinsed with distilled water. The cellulose II sample was obtained by mercerization of MC.

\section{$X$-Ray Measurements}

To characterize the structure of the product from Acetobacter-culture in the presence of DR75 or DR79 (DR75 and DR79 products), first an uniplanar-oriented membrane was prepared from the preserved product, which was dried on a teflon plate at atmospheric conditions, for reflection and transmission. The diffractograms of the isotropic product samples and cellulose regenerated from it were obtained by transmission. The sample was compressed as a pellet to $2 \mathrm{~mm}$ diameter and $1 \mathrm{~mm}$ thick pore of a sample holders. An X-ray diffractogram was obtained by a $\mathbf{M X P}^{18}$ diffractometer (MAC Science) with $\mathrm{Ni}$-filtered $\mathrm{Cu}-K_{\alpha}$ radiation. The measurement conditions were as followed: divergence slit, $1.0 \mathrm{~mm}$; receiving slit, $0.15 \mathrm{~mm}$; scanning speed, $4.0^{\circ} \mathrm{min}^{-1}$; X-ray radiation, $40 \mathrm{kV}, 200 \mathrm{~mA}$.

\section{Solid State ${ }^{13} C$ NMR Measurements}

Solid state ${ }^{13} \mathrm{C}$ NMR of the wet samples was performed at room temperature on a JEOL JNM EX 270 spectrometer operating at $6.35 \mathrm{~T}$, as described elsewhere. ${ }^{1-4}$ MAS rates were $5.0-5.5 \mathrm{kHz}$ and the chemical shifts relative to tetramethylsilane were determined by using the crystalline peak at $17.3 \mathrm{ppm}$ of hexamethyl benzene as an external standard. The non-dried samples were packed in MAS rotor with an O-ring sealed to avoid the loss of water during NMR measurements. To determine the crystal structure of regenerated cellulose, solid state ${ }^{13} \mathrm{C}$ NMR was measured by Torchia sequence $(\tau=50 \mathrm{~s}) .^{5}$

\section{Deuteration-Infrared Measurements}

To obtain IR spectra of the sample, a membrane of about $10 \mu \mathrm{m}$ in dry thickness was prepared from the non-dried preserved samples. The deuteration of the sample was performed by the method in the previous paper. ${ }^{1-4}$ The sample membrane was set inside a glass cell so that the IR beam was perpendicular to the membrane surface. The glass cell which contained the sample was dried under reduced pressure $\left(10^{-3}\right.$ Torr $)$ for $2 \mathrm{~h}$, and then vapor phase deuteration of the sample was performed by $\mathrm{D}_{2} \mathrm{O}$ (purity of $99.8 \%$; Aldrich Chemical Co.) for a specified time at room temperature. It was again dried under reduced pressure, and the IR spectrum was measured. FT-IR system 800 (Nicolet) was used for the IR spectroscopy. OH and OD bands were calculated according to the method described in the previous paper. ${ }^{1-4}$

\section{RESULTS}

\section{$X$-Ray Diffractograms of the Products}

Figure 2 shows the X-ray diffractograms of the isotropic sample of MC, DR75, and DR79 products. As

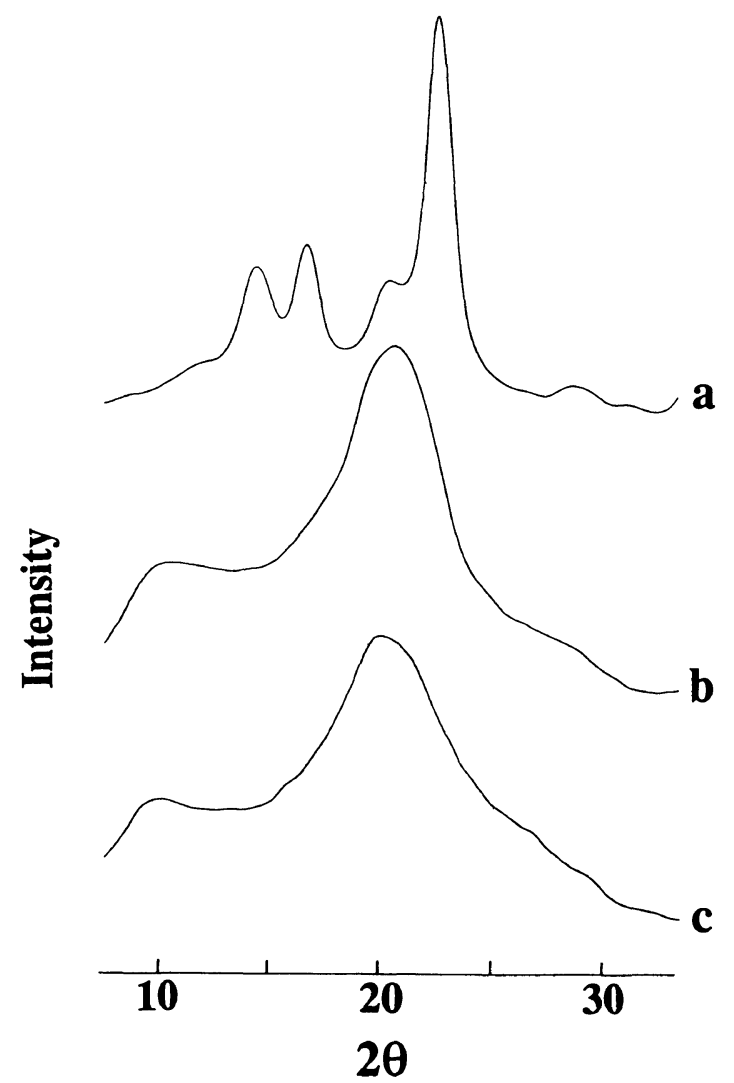

Figure 2. X-Ray diffractograms of microbial cellulose and the product samples from Acetobacter-culture in the presence of a dye of $0.1 \mathrm{wt} \%$. a, MC; b, DR75 product; c, DR79 product.

Table I. X-Ray diffraction angles of the product sample

\begin{tabular}{lccccc}
\hline Sample & \multicolumn{5}{c}{$2 \theta / \mathrm{deg}$} \\
\hline MC & - & $14.5(\mathrm{w})$ & $16.8(\mathrm{w})$ & $20.4(\mathrm{w})$ & $22.7(\mathrm{~s})$ \\
DR75 & $10.1(\mathrm{w})$ & - & - & $20.6(\mathrm{~s})$ & - \\
DR79 & $10.1(\mathrm{w})$ & - & - & $19.9(\mathrm{~s})$ & - \\
\hline
\end{tabular}

apparent from Figure 2, the diffraction patterns of the products are different from that of MC. The diffraction peaks of the (110), (110), and (020) planes of MC occur at $2 \theta: 14.5,16.8$, and $22.7^{\circ}$ ( Table I). In contrast, in the diffractograms of each DR75 and DR79 product, there are no peaks at (110), (110), and (020) plane position of MC. Two new diffraction peaks are observed in the diagram of products. The lower angle peak appears near $10^{\circ}$, while the higher angle peak near $20^{\circ}$. The diffractograms of DR75 and DR79 products are similar to those of DB1, DB14, DB15, and DB53 products as reported in the previous papers. ${ }^{1-4}$ It is clear that the product, formed by the interaction of dye and cellulose chain, is a crystalline dye-cellulose complex. In the dye-cellulose complex, the hydrogen bonding of MC is hindered by the dye molecule included between the cellulose sheets. The dye molecule binds the cellulose chains through the intermolecular hydrogen bonding and van der Waals forces. ${ }^{1-4}$

As apparent from Figure 3, if measured with either transmission or reflection method, the diffractograms of each membrane of the DR75 and DR79 products are similar to each other. The uniplanar-oriention of these product membranes are not similar to that of DB14 and 


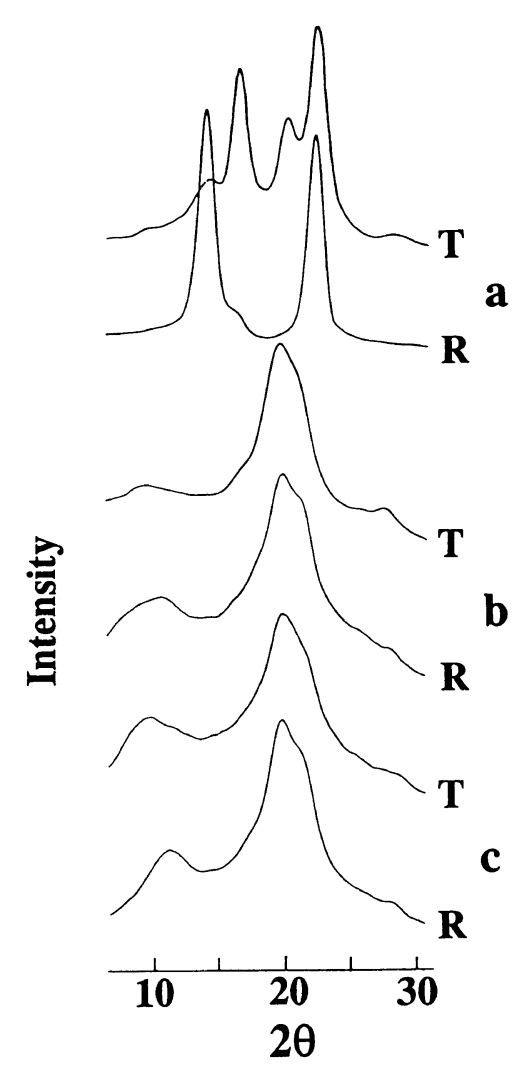

Figure 3. X-ray diffractograms of microbial cellulose and the product samples measured by transmission ( $T$ ) and reflection (R) methods. a, MC; b, DR75 product; c, DR79 product.

DB15 product membranes. ${ }^{4}$ As the diffraction diagram of the membrane of DR75 and DR79 products measured by either reflection or transmission method does not change its pattern and the pattern is similar to that of their isotropic sample, the DR75 and DR79 product membranes do not attain uniplanar orientation. Therefore, the uniplanar orientation of these samples suggests that the interaction of dye and cellulose are somewhat different to that of the dyes, having biphenylenebis(azo) skeletal backbone, and cellulose. It is not clear whether the uniplanar orientation of DR75 and DR79 products is affected by the structure of dyes or ureylene group or both.

\section{$X$-Ray Diffractograms of Cellulose Regenerated from the Products}

The X-ray diffractograms of cellulose regenerated from the DR75 and DR79 products ( DR75 and DR79 celluloses ) and cellulose II are shown in Figure 4 and $2 \theta$ of diffraction peaks of each sample are listed in Table II.

From Figure 4 and Table II, X-ray diffractograms of DR75 and DR79 celluloses show the typical diffraction patterns of cellulose II (mercerized MC). These diffractograms are similar to those of celluloses regenerated from the product in the presence of DB1, DB14, DB15, or DB53. ${ }^{3,4}$ Although it is assumed that the affinity of the dye having ureylenebis(naphthylazo) benzene skeletal structure toward cellulose chains is stronger than that of the dye having biphenylenebis(azo) skeletal structure because of ureylene group in different skeletal structure, X-ray measurement suggests that the

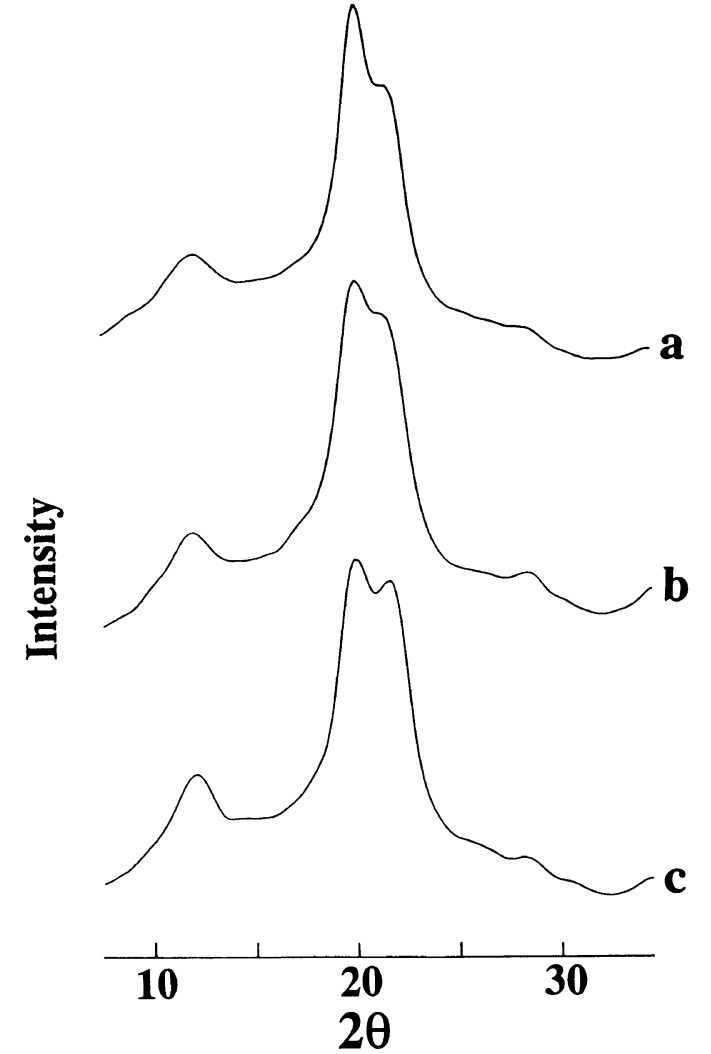

Figure 4. X-Ray diffractograms of the celluloses regenerated from the product samples. a, cellulose II; b, DR75 cellulose; c, DR79 cellulose.

Table II. X-Ray diffraction angles of the regenerated cellulose samples

\begin{tabular}{cccc}
\hline & \multicolumn{3}{c}{$20 /$ deg } \\
\cline { 2 - 4 } Sample & $(1 \overline{1} 0)$ & $(110)$ & $(020)$ \\
\hline DR75 & 12.1 & 20.0 & 21.8 \\
DR79 & 12.2 & 20.0 & 21.7 \\
Cellulose II & 12.0 & 19.8 & 21.1 \\
\hline
\end{tabular}

enlargement of affinity of the dye toward cellulose influence the uniplanar orientation of the product and the fine structure of cellulose regenerated from it.

\section{Solid State ${ }^{13} C$ NMR Spectra of MC, Product and Dye Powder}

Figure 5 shows the solid state ${ }^{13} \mathrm{C}$ NMR spectra of MC, DR75, DR79 products and corresponding dye powders. In Table III, the chemical shifts of resonance lines of MC and cellulose component of DR75 and DR79

In Figure 5 the resonance lines of cellulose component in the DR75 product do not overlap the lines of the corresponding DR75 powder. In the case of DR79 product, the upfiled side of C6 and C2, 3, 5 resonance lines and part of $\mathrm{Cl}$ resonance line overlap the lines of dye powder. But ${ }^{13} \mathrm{C}$ NMR of both products are similar to each other and different to that of MC. The ratio of to each other and different to that of MC. The ratio of the dye to cellulose in the DR79 product is not high. Besides the pattern of resonance lines, as apparent in Table III, there are no differences between the chemical shifts of DR75 and DR79 products. It is possible to 


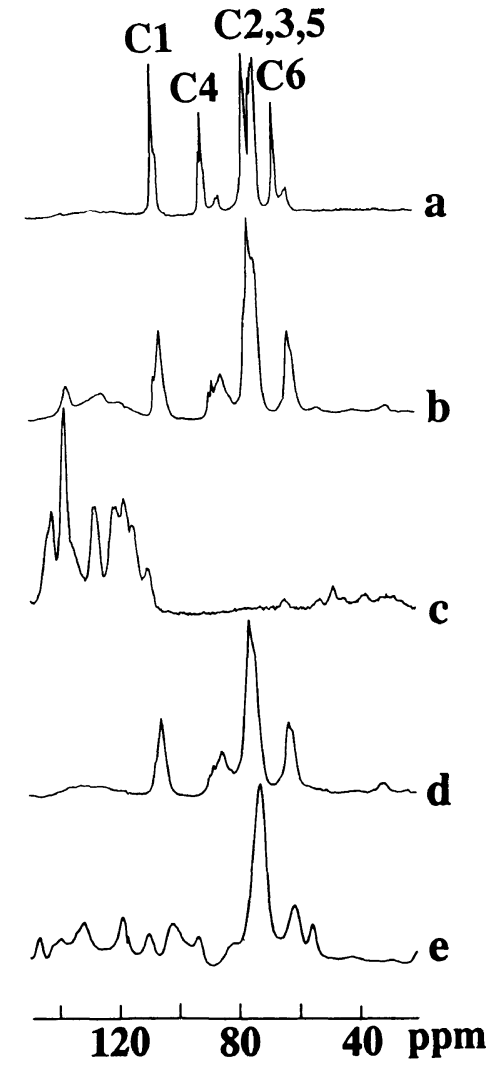

Figure 5. Solid state ${ }^{13} \mathrm{C}$ NMR spectra of the wet MC, product samples and dry direct dyes. a, MC; b, DR75 product; c, DR75 powder; d, D79 product; e, DR79 powder.

Table III. ${ }^{13} \mathrm{C}$ NMR chemical shifts (ppm) of product samples

\begin{tabular}{|c|c|c|c|c|}
\hline Sample & $\mathrm{Cl}$ & $\mathrm{C} 4$ & $\mathrm{C} 2,3,5$ & C6 \\
\hline $\mathrm{MC}$ & 106.3 & $90.2,84.7$ & $75.9,73.8,72.3$ & $66.5,62.5$ \\
\hline DR75 & 105.4 & $-\quad 85.0$ & $75.3--$ & - 62.8 \\
\hline DR79 & 105.6 & 85.2 & 75.5 & -63.1 \\
\hline
\end{tabular}

discuss the structure of the cellulose component in the DR75 and DR79 products.

The resonance lines of each carbon of the cellulose component in the products are broader than those of MC. As apparent in Figure 5 and Table III, the chemical shifts of the resonance lines of $\mathrm{C} 4$ and $\mathrm{C} 6$ are at near the 84.7 and $62.5 \mathrm{ppm}$ which are the chemical shifts of noncrystalline component of MC. The two small peaks at near $\mathrm{C} 4,89.5$ and $88.5 \mathrm{ppm}$, respectively, may be the resonance lines of cellulose II in the products. However, the facts suggest that the whole spectra of cellulose component in the product is in the noncrystalline state.

\section{Solid State ${ }^{13}$ C NMR Spectra of Cellulose Regenerated from Products and Cellulose II}

Figure 6 shows the solid state ${ }^{13} \mathrm{C}$ NMR spectra of crystalline component of DR75 and DR79 celluloses and cellulose II. The chemical shifts of the resonance lines of regenerated cellulose and cellulose II are listed in Table IV.

As apparent from Figure 6 and Table IV, the spectra of the crystalline component in the DR75 and DR79 celluloses and corresponding chemical shifts of $\mathrm{Cl}, \mathrm{C} 4$, and C6 lines indicate that the DR75 and DR79 celluloses

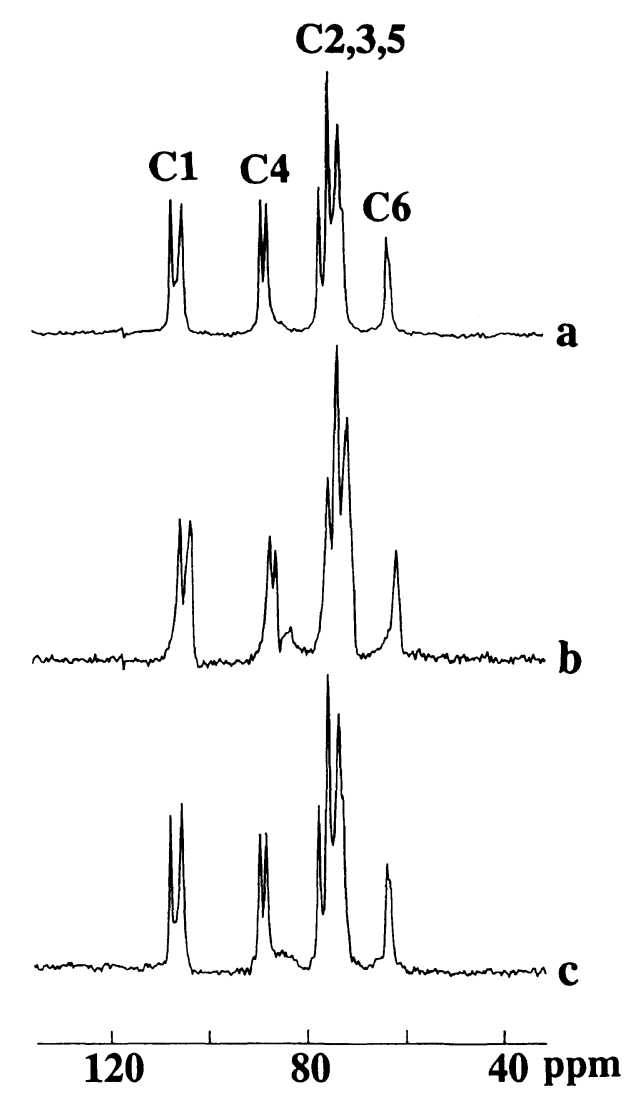

Figure 6. Solid state ${ }^{13} \mathrm{C}$ NMR spectra of crystalline components of the wet cellulose regenerated from the products. a, cellulose II; b, DR75 cellulose; c, DR79 cellulose.

Table IV. ${ }^{13} \mathrm{C}$ NMR chemical shifts (ppm) of the crystalline component of regenerated celluloses and cellulose II

\begin{tabular}{ccccc}
\hline Sample & C1 & C4 & C2, 3, 5 & C6 \\
\hline DR75 & $107.8,105.7$ & $89.5,88.3$ & $77.4,75.6,73.7$ & 63.7 \\
DR79 & $107.9,105.6$ & $89.5,88.2$ & $77.3,75.6,73.4$ & 63.7 \\
Cellulose II & $107.5,105.3$ & $89.3,87.9$ & $77.0,75.2,72.9$ & 63.2
\end{tabular}

are cellulose II. The ratio of the strength of downfield C1 resonance line to that of upfield side of DR75 and DR79 celluloses is larger than that of cellulose regenerated from the DB1, DB14, DB15, and DB53 products. ${ }^{1-4}$ The downfield peak of the $\mathrm{C} 1$ resonance line indicates the situation of hydrogen bond formation. ${ }^{6,7}$ The sharpness and relative abundance of this signal suggests that the cellulose II structure of DR75 and DR79 celluloses is more stable than that of DB1, DB14, DB15, and DB53 celluloses. ${ }^{1-4}{ }^{13} \mathrm{C}$ NMR of DR75 and DR79 celluloses have good agreement with that of X-ray measurements.

\section{IR Spectra of Products by Vapor Phase Deutration at}

\section{Room Temperature}

Change in the IR spectra of $\mathrm{OH}$ and OD bands in the DR75 and DR79 products by vapor phase deuteration at room temperature is shown in Figure 7 and in Table $\mathrm{V}$, the ratio of accessible $\mathrm{OH}$ group (OD group) and nonaccessible $\mathrm{OH}$ group are listed.

Nonaccessible OH groups of DR75 and DR79 products are $29 \%$ and $28 \%$, respectively. These nonaccessible $\mathrm{OH}$ bands show the spectra having the cha- 


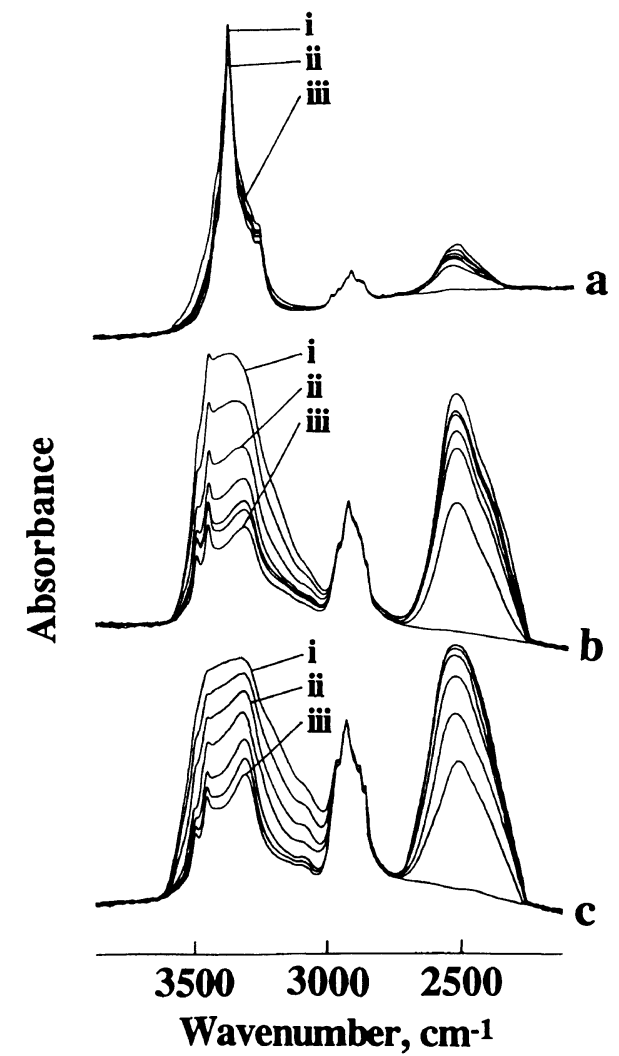

Figure 7. Changes in IR spectra of the product samples by vapor phase deuteration at room temperature. a, MC; b, DR75 product; c, DR79 product. Deuteration time (min): i, 0; ii, 3; iii, 1000.

Table V. Amount of $\mathrm{OH}$ and $\mathrm{OD}$ bands of the product and MC

\begin{tabular}{lcc}
\hline \multicolumn{1}{c}{ Sample } & OH band $/ \%$ & OD band $/ \%$ \\
\hline MC & 85 & 15 \\
DR75 product & 29 & 71 \\
DR79 product & 28 & 72 \\
\hline
\end{tabular}

${ }^{a}$ Nonaccessible $\mathrm{OH}$ band.

racteristic structure of cellulose II. As is described previously, ${ }^{1-4}$ the nonaccessible $\mathrm{OH}$ groups seem to occur in cellulose rich region of the product sample due to rinsing during the preparation process. The characteristics of these $\mathrm{OH}$ bands of the products are that the absorption of intermolecular hydrogen bonding occur at near $3300 \mathrm{~cm}^{-1}$ instead of at near $3340 \mathrm{~cm}^{-1}$ of cellulose II. Since the absorptions of intermolecular hydrogen bonding due to nonaccessible $\mathrm{OH}$ groups of DB1, DB14, DB15, and DB53 products occur at 3340 $\mathrm{cm}^{-1,1-4}$ DR75 and DR79 must have somewhat different action foward nascent cellulose chains. It is assumed that the stronger intermolecular hydrogen bonding between the dye and cellulose chains are due to the ureylene groups in the dye.

IR Spectra of Cellulose Regenerated from Products by Vapor Phase Deuteration at Room Temperature

Figure 8 shows change in the IR spectra of $\mathrm{OH}$ and OD bands in DR75 and DR79 celluloses by vapor phase deuteration at room temperature. The ratio of accessible $\mathrm{OH}$ group and nonaccessible $\mathrm{OH}$ groups is listed in Table VI.

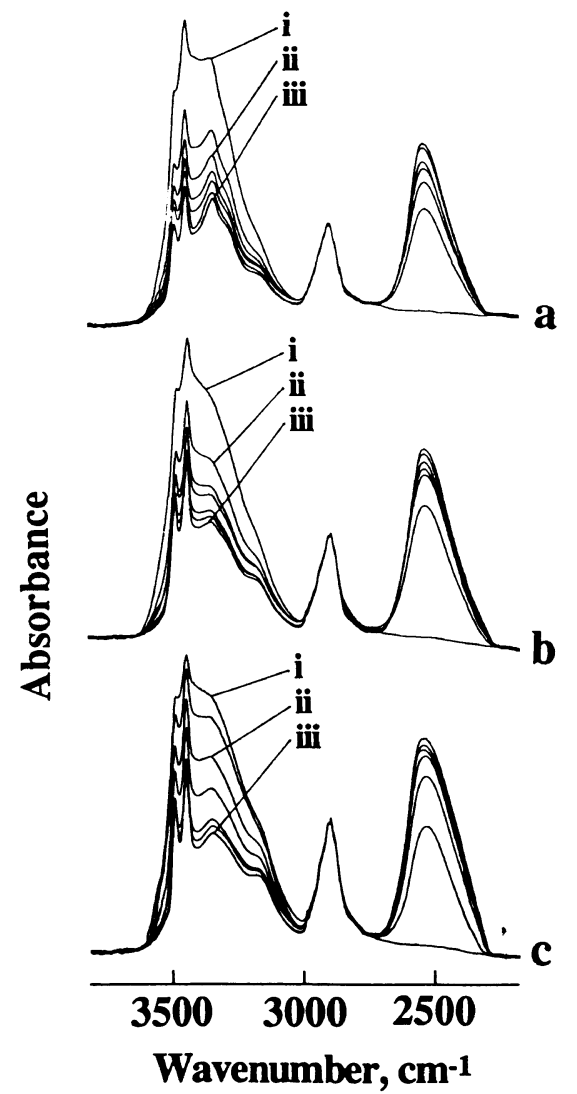

Figure 8. Changes in IR spectra of celluloses regenerated from the product samples by vapor phase deuteration at room temperature. a, cellulose II; b, DR75 cellulose; c, DR79 cellulose. Deuteration time (min): i, 0; ii, 3; iii, 1000.

Table VI. Amount of $\mathrm{OH}$ and $\mathrm{OD}$ bands of regenerated cellulose

\begin{tabular}{lcc}
\hline Sample & $\mathrm{OH} \mathrm{band}^{\mathrm{a}} / \%$ & OD band $/ \%$ \\
\hline Cellulose II & 45 & 55 \\
DR75 cellulose & 48 & 52 \\
DR79 cellulose & 45 & 55
\end{tabular}

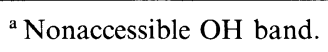

Nonaccessible $\mathrm{OH}$ group of DR75 and DR79 celluloses are $48 \%$ and $45 \%$, respectively. The nonaccessible $\mathrm{OH}$ groups show the spectra of the typical cellulose II which has two absorptions by intramolecular hydrogen bonding at near $3485 \mathrm{~cm}^{-1}$ and $3443 \mathrm{~cm}^{-1}$, and two absorptions by intermolecular hydrogen bonding at near $3340 \mathrm{~cm}^{-1}$ and $3174 \mathrm{~cm}^{-1} .8$ These hydrogen bondings of regenerated cellulose strongly support the $\mathrm{X}$-ray and ${ }^{13} \mathrm{C}$ NMR measurements. More nonaccessible OH groups in DR75 and DR79 celluloses compared to that of DB1, DB14, DB15, and DB53 celluloses ${ }^{3,4}$ suggests that the crystallinity of the former group is higher, and supports the more stable hydrogen bond formation concept in the complex.

\section{DISCUSSION}

Although all substituent groups are not common and the position of sulfonate groups are not the same, there are no differences between the structure of the products obtained in the presence of DR75 and DR79 having 
ureylenebis(naphthylazo)benzene skeletal structure. Judging from X-ray measurement results of the products, these products show the diffractograms having two peaks at near 10 and $20^{\circ}$ and the diffraction patterns are similar to those of the products in the presence of DB1, DB14, DB15, and DB53 having biphenylenebis(azo) skeletal structure. $^{1-4}$ However, in the membranes of DR75 and DR79 products, uniplanar-orientation is not observed whereas the membranes of the DB1, DB14, DB15, and DB53 products showed weak uniplanarorientation in comparison with DR28 and MC. ${ }^{1-4,9}$ Solid state ${ }^{13} \mathrm{C}$ NMR measurements indicate that both products in the presence of DR75 and DR79 are the noncrystalline complex which is contrasted to X-ray measurement results. In the X-ray measurements, the product is a crystalline complex. However, DR75 and DR79 hinder normal hydrogen bonding of MC in the complex by their inclusion in as a monomolecular layer between the cellulose sheets corresponding to the (110) plane of MC. ${ }^{9}$ This is also supported by deuterationIR measurements. Accessible OH group of DR75 and DR79 products increases to $71 \%$ and $72 \%$, respectively, compared to that of $15 \%$ of MC. The IR spectra of the nonaccessible $\mathrm{OH}$ groups of the products show the spectra of cellulose II. This suggests that the weaker bonded dye from the product is washed off during rinsing and this washed off region in the products appears as nonaccessible $\mathrm{OH}$ groups. The dye molecule between the cellulose sheets or chains in the product affect the conformation of the cellulose chains during rinsing due to which the structure of MC transforms to cellulose II structure in the cellulose rich portion in the product. This change also occurs in the regenerated cellulose due to the same reasons. But the higher crystallinity and more stable hydrogen bond formation in DR75 and DR79 celluloses than that of DB1, DB14, DB15, and DB53 celluloses ${ }^{3,4}$ are the cause of ureylene groups. The structures of product as well as regenerated cellulose are not affected by the uncommon methyl, methoxy, or amino groups.

All measurements, i.e., X-ray, the solid state ${ }^{13} \mathrm{C}$ NMR, and deuteration-IR results indicate that celluloses regenerated from DR75 and DR79 products form the typical structure of cellulose II. The resonance line of $\mathrm{C} 1$ in the spectra of ${ }^{13} \mathrm{C}$ NMR indicates that both regenerated celluloses form the stable structure of cellulose II.

The main influence of the dye having ureylenebis(naphthylazo)benzene skeletal structure and four sulfonate groups, and the dye also having four sulfonate groups, and biphenylenebis(azo) skeletal structure on the structures of the product and cellulose regenerated from it is almost the same, that is, all products are noncrystalline and all regenerated celluloses are cellulose II, except for DR28 which having only two sulfonate groups. The differences of effects of DR28 and other direct dyes on the nascent structure of MC seem to be mainly due to the number of sulfonate groups per unit molecular weight in the dye. This suggests that only sulfonate groups in a dye affect the conformation of nascent MC to transform other cellulose allomorphs, but other substituent groups affect conformation in the cellulose allomorph which is already affected by the sulfonate groups.

\section{CONCLUSION}

The influence of Direct Red 75 and 79 having the same ureylenebis(naphthylazo) benzene skeletal structure on the nascent structure of microbial cellulose was examined. Each product obtained from Acetobacter-culture in the presence of Direct Red 75 or Direct Red 79 is a crystalline complex in which the monolayer of a dye is included between the cellulose sheets corresponding to the (110) plane of microbial cellulose. Cellulose regenerated from each product forms cellulose II. Although the substituent groups in the skeletal structure of both the dyes are not the same, their interactions on the nascent structure of microbial cellulose are almost the same. Due to the ureylene group on the skeletal structure of dye, stronger hydrogen bonding occurrs between the dye and cellulose in the product. The ureylene group also affect to form the stable hydrogen bonding in the cellulose regenerated from Direct Red 75 and Direct Red 79 products.

\section{REFERENCES}

1. A. Kai and Md. I. H. Mondal, Int. J. Biol. Macromol., 20, 221 (1997)

2. Md. I. H. Mondal and A. Kai, Int. J. Biol. Macromol., in press.

3. Md. I. H. Mondal and A. Kai, Polym. J., 30, 78 (1998).

4. Md. I. H. Mondal and A. Kai, Polym. J., 30, 84 (1998).

5. D. A. Torchia, J. Magn. Reson., 30, 613 (1978).

6. R. L. Dudley, C. A. Fyfe, P. J. Stephenson, Y. Deslandes, G. K. Hamer, and R. H. Marchessault, J. Am. Chem. Soc., 105, 2469 (1983).

7. F. Horii, A. Hirai, and R. Kitamaru, Polym. Bull., 8, 163 (1982).

8. S. Okajima and A. Kai, J. Polym. Sci., A-1, 6, 2801 (1968).

9. A. Kai and Md. I. H. Mondal, in "Biomedical Functions and Biotechnology of Natural and Artificial Polymers," M. Yalpani, Ed., ATL Press, Inc., Science Publishers, Shrewsbury, MA, 1996, p 77. 